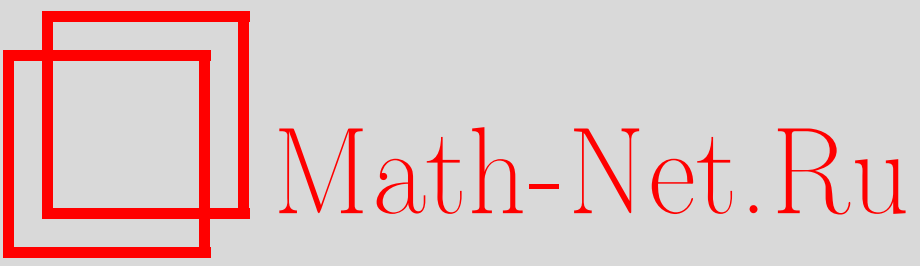

В. В. Балащенко, Однородные эрмитовы $f$-многообразия, УMH, 2001, том 56 , выпуск 3, 159-160

DOI: https://doi.org/10.4213/rm401

Использование Общероссийского математического портала Math-Net.Ru подразумевает, что вы прочитали и согласны с пользовательским соглашением

http://www.mathnet.ru/rus/agreement

Параметры загрузки:

IP : 18.234 .197 .8

26 апреля 2023 г., 15:18:43 


\title{
ОДНОРОДНЫЕ ЭРМИТОВЫ $f$-МНОГООБРАЗИЯ
}

\author{
В. В. БАЛАЩЕНКО
}

Инвариантным структурам традиционно (и заслуженно) принадлежит ведущая роль при исследовании дифференциально-геометрических структур на гладких многообразиях. Именно благодаря широкому классу инвариантных примеров был получен ряд глубоких результатов в период бурного развития эрмитовой геометрии (см., например, [1]-[3]), причем поток исследований здесь не ослабевает и сейчас. Наряду с этим изучение почти контактных структур и их приложений, $f$-структур К. Яно $\left(f^{3}+f=0\right)$ и других обобщений привело к созданию в 80 -х годах в работах В. $\Phi$. Кириченко стройной концепции обобщенной эрмитовой геометрии (см., например, [4]). Число исследований в этом новом направлении стремительно растет, причем в различных аспектах (см., например, [5]), а потому все ощутимее становится отсутствие инвариантных примеров.

Ситуация качественно изменилась в последнее время в связи с полным решением проблемы описания канонических структур классического типа на регулярных $\Phi$-пространствах [6]. Был обнаружен богатый запас канонических $f$-структур (в том числе почти комплексных структур), которые позволили предъявить болшшие классы инвариантных примеров в обобщенной эрмитовой геометрии (см. [7], [8]), что обеспечило преемственность классических результатов Дж. Вольфа и А. Грея в эрмитовой геометрии.

Алгебраической основой выделения важнейших классов обобщенных почти әрмитовых структур являются свойства присоединенной $Q$-алгебры [4]. В случае метрической $f$-структуры композиционный тензор $T$ вычислен в [4] явно:

$$
4 T(X, Y)=f\left(\nabla_{f X}(f) f Y-\nabla_{f^{2} X}(f) f^{2} Y\right),
$$

где $X, Y$ - векторные поля на $M$. Антикоммутативность $Q$-алгебры $(T(X, X)=0)$ определяет обобщенные $G_{1}$-структуры, а ее абелевость $(T(X, Y)=0)$ - обобщенные әрмитовы струк$m y p b$. Для случая метрической $f$-структуры будем назьвать их $G_{1} f$-структурой и эрмитовой $f$-структурой соответственно. Очевидно, что в частном случае $f=J\left(J^{2}=-1\right)$ получаем в точности классические понятия $G_{1}$-структуры и эрмитовой структуры (см., например, $\left.[9]\right)$. При этом эрмитова $f$-структура, в отличие от классического случая, не всегда интегрируема.

В данной заметке указаны обширные классы инвариантных $G_{1} f$-структур и эрмитовых $f$ структур, при этом особую роль играют канонические $f$-структуры на однородных $\Phi$-пространствах порядка $k$ ( $k$-симметрических пространствах в терминологии [10]).

Пусть $(G / H, g, f)$ - однородное редуктивное пространство группы Ли $G$ с инвариантной (псевдо-) римановой метрикой $g$ и инвариантной метрической $f$-структурой, $\mathfrak{g}=\mathfrak{h} \oplus \mathfrak{m}$ - соответствующее редуктивное разложение алгебры Ли $\mathfrak{g}$ группы $G$. Условимся не различать в обозначениях инвариантные структуры на $G / H$ и их значения в точке $o=H$. Структура $f$ порождает ортогоналшное разложение $\mathfrak{m}=\mathfrak{m}_{1} \oplus \mathfrak{m}_{2}$, где подпространства $\mathfrak{m}_{1}=\operatorname{Im} f$ и $\mathfrak{m}_{2}=\operatorname{Ker} f$ определяют соответственно первое и второе фундаментальные распределения для $f$. Напомним, что $(G / H, g)$ назьвается естеств енно редуктивныц, если $g\left([X, Y]_{\mathfrak{m}}, Z\right)=g\left(X,[Y, Z]_{\mathfrak{m}}\right)$ для всех $X, Y, Z \in \mathfrak{m}$, где индекс $\mathfrak{m}$ обозначает проекцию на $\mathfrak{m}$ относительно редуктивного разложения.

Теорема 1. Любая инвариантная метрическая $f$-структура на естественно редуктивном пространстве $(G / H, g)$ является $G_{1} f$-структурой.

Как частный случай, отсюда получаем

СлЕДСТВИЕ. На естественно редуктивном пространстве $(G / H, g)$ любая инвариантная почти эрмитова структура принадлежит классу $G_{1}$.

Отметим, что это утверждение по эрмитовой геометрии получено в работе [11].

Теорема 2. Пусть $(G / H, g, f)$ - естественно редуктивное пространство с инвариантной метрической $f$-структурой, для которой выполняется условие $\left[\mathfrak{m}_{1}, \mathfrak{m}_{1}\right] \subset$ $\mathfrak{m}_{2} \oplus \mathfrak{h}$. Тогда $G / H$ - эрмитово $f$-многообразие.

Далее будем рассматривать регулярные $\Phi$-пространства (обобщенные симметрические пространства [10]), на которых имеется значительный запас канонических структур $f$ и $J$ [6]. 
Пусть $G / H$ - регулярное $\Phi$-пространство, определяемое автоморфизмом $\Phi$ связной группь Ли $G, \mathfrak{g}=\mathfrak{h} \oplus \mathfrak{m}$ - каноническое редуктивное разложкение, соответствующее автоморфизму $\varphi=d \Phi_{e}$ алгебры Ли $\mathfrak{g}$, т.е. $\mathfrak{m}=A_{\varphi} \mathfrak{g}$, где $A_{\varphi}=\varphi-\mathrm{id}[12]$. Обозначим через $\theta$ сужение $\varphi$ на $\mathfrak{m}$. Инвариантная афффинорная структура $F$ на $G / H$ назьвается канонической [6], если она в точке $о$ является полиномом $F=F(\theta)$. Все однородные $\Phi$-пространства порядка $k\left(\Phi^{k}=\mathrm{id}\right)$ регулярны [12], и для таких пространств в [6] получены формулы для всех канонических структур типов $f$ и $J$. Всюду в дальнейшем под естественно редуктивным разложением подразумевается каноническое редуктивное разложение для $G / H$. Например, для полупростой группы $G$ метрика $g$, порождаемая формой Киллинга на любом регулярном $\Phi$-пространстве $G / H$, естественно редуктивна (см. [12]). Важно отметить, что все канонические структуры $f$ и $J$ с такой метрикой согласованы [7].

Теорема 3. Пусть $(G / H, g)$ - естественно редуктивное однородное Ф-пространство порядка $k$. Любая каноническая метрическая $f$-структура на $G / H$ является $G_{1} f$-структурой, а любая каноническая почти әрмитова структура $J$ входит $в$ класс $G_{1}$.

Отметим особую роль однородных $\Phi$-пространств порядков 4 и 5:

Теорема 4. Естественно редуктивное $\Phi$-пространство $(G / H, g, f)$ порядка 4, где $f=\frac{1}{2}\left(\theta-\theta^{3}\right)-$ каноническая метрическая $f$-структура, является эрмитовым $f$-многообразием.

Теорема 5. Пусть $(G / H, g)$ - естественно редуктивное Ф-пространство порядка 5 , $f_{1}$ и $f_{2}$ - канонические метрические $f$-структурь на $G / H$ (cм. [6]). Тогда $\left(G / H, g, f_{i}\right)$, $i=1,2$, является әрмитовым $f$-многообразием.

Таким образом, указанные в теоремах 4 и 5 канонические $f$-структуры обеспечивают широкий класс первых примеров инвариантных эрмитовых $f$-структур, которые в общем случае не являются интегрируемьми. Более того, имеющаяся деталшная классификация однородных $\Phi$-пространств порядков 4 и 5 классических компактных групп Ли (см. [13], [14]) позволяет поэтому предъявить явную серию однородных эрмитовых $f$-многообразий.

Известньм примером риманова однородного $\Phi$-пространства порядка 4 , метрика которого не является естественно редуктивной, служит 6-мерная обобщенная группа Гейзенберга (см. [15]).

Теорема 6. 6-мерная обобщенная группа Гейзенберга $(N, g)$ относительно канонической $f$-структуры однородного Ф-пространства порядка 4 является однородным әрмитовым $f$-многообразием.

\section{СПИСОК ЛИТЕРАТУРЫ}

[1] J. Wolf, A. Gray // J. Differential Geom. 1968. V. 2. №1-2. P. 77-159. [2] A. Gray // J. Differential Geom. 1972. V. 7. № 3-4. Р. 343-369. [3] В. Ф. Кириченко // Матем. заметки. 1981. Т. 30. № 4. С. 569-582. [4] В.Ф. Кириченко // Итоги науки и техники. Проблемы геометрии. Т. 18. М.: ВИНИТИ, 1986. С. 25-71. [5] В. Ф. Кириченко, Л. В. Липагина // Изв. РАН. Сер. матем. 1999. Т. 63. № 5. С. 127-146. [6] В. В. Балащенко, Н. А. Степанов // Матем. сб. 1995. T. 186. №11. C. 3-34. [7] V. V. Balashchenko. Riemannian geometry of canonical structures on regular $\Phi$-spaces // Preprint № 174/1994: Ruhr-Universität Bochum, 1994. [8] В. В. Балащенко // УМН. 1999. Т. 54. № 3. С. 151-152. [9] A. Gray, L. M. Hervella // Ann. Mat. Pura Appl. (4). 1980. V. 123. № 4. P. 35-58. [10] О. Ковальский. Обобщенные симметрические пространства. М.: Мир, 1984. [11] E. Abbena, S. Garbiero // Nihonkai Math. J. 1993. V. 4. № 1. P. 1-15. [12] Н. А. Степанов // Изв. вузов. Матем. 1967. № 3. С. 88-95. [13] J. A. Jimenez // Trans. Amer. Math. Soc. 1988. V. 306. № 2. P. 715-734. [14] Gr. Tsagas, Ph. Xenos // Bull. Math. Soc. Sci. Math. R. S. Roumanie (N.S.). 1987. V. 31. № 1. P. 57-77. [15] F. Tricerri, L. Vanhecke. Homogeneous Structures on Riemannian Manifolds. Cambridge: Cambridge Univ. Press, 1983. (London Math. Soc. Lecture Note Ser. V. 83.) 